\title{
Biofilm Growth and Fossil Form
}

\author{
A. P. Petroff* \\ Lorenz Center and Department of Earth, Atmospheric, and Planetary Sciences, \\ Massachusetts Institute of Technology, Cambridge, Massachusetts 02139, USA
}

N. J. Beukes

Department of Geology, University of Johannesburg, Post Office Box 524, Auckland Park 2006, Kingsway and University (APK Campus), Johannesburg, South Africa

D. H. Rothman

Lorenz Center and Department of Earth, Atmospheric, and Planetary Sciences, Massachusetts Institute of Technology, Cambridge, Massachusetts 02139, USA

T. Bosak

Department of Earth, Atmospheric, and Planetary Sciences, Massachusetts Institute of Technology, Cambridge, Massachusetts 02139, USA

(Received 9 August 2012; revised manuscript received 6 June 2013; published 13 November 2013)

Stromatolites can grow under the influence of microbial processes, but it is often unclear whether and how the macroscopic morphology of these rocks records biological processes. Conical stromatolites, which formed in the absence of sedimentation, provide a comparatively simple record of the interplay between microbial growth and lithification. Here, we show that the dynamics shaping conical stromatolites result from diffusive gradients within the overlying microbial mat. These gradients cause minerals to precipitate faster in regions of high curvature, resulting in measurable properties of the shapes of stromatolite laminas. This model allows us to estimate the thickness of ancient stromatolite-forming mats to be approximately $1 \mathrm{~mm}$, consistent with modern systems. Proceeding from the assumption that the ubiquitous process of diffusion is recorded in the translating form of a stromatolite, we derive the shape of a diffusion-driven stromatolite. The conical morphology-a distinctive feature of stromatolites growing in the absence of sedimentation-arises from these dynamics. This form is quantitatively consistent with the shape of conical stromatolites that grew for more than $2.9 \times 10^{9}$ yrs of Earth history.

DOI: 10.1103/PhysRevX.3.041012

\section{INTRODUCTION}

Laminated, lithified sedimentary rocks called stromatolites are commonly regarded as Earth's earliest macroscopic fossils [1-3]. The formation of petrified stromatolites involves mineral precipitation and sedimentation that can be influenced by microbial activity [4,5]. The resulting morphology of a stromatolite is therefore a consequence of the coupling between biotic processes such as mat growth and abiotic processes such as mineralization [3]. Given this interpretation of stromatolite growth, it is natural to ask what, if any, biological information is recorded in the shape of a stromatolite [6-8].

Much recent work has focused on understanding the growth of a particularly simple class of stromatolites that develops a conical morphology [8-11], examples of which

\footnotetext{
*Present address: Center for Studies in Physics and Biology, The Rockefeller University, New York, NY 10021, USA.

Published by the American Physical Society under the terms of the Creative Commons Attribution 3.0 License. Further distribution of this work must maintain attribution to the author(s) and the published article's title, journal citation, and DOI.
}

Subject Areas: Biological Physics, Interdisciplinary Physics

are shown in Fig. 1. Because conical stromatolites typically grew in quiet, sediment-poor conditions, this morphology is one of the dynamically simplest types of stromatolites $[5,12,13]$. This form has persisted over approximately three-fourths the age of Earth $[1,6,10,11,14,15]$.

Shall we conclude from this startling uniformity of form that the biological and environmental processes shaping conical stromatolites have been similarly constant? In this paper, we suggest an alternative possibility. The geometry of a conical stromatolite does not reflect the influence of any single biological or environmental factor but rather a geometric feature of the dynamics common to a class of processes. Here, we show that this class is defined by diffusion through a thin film.

Understanding the growth dynamics of rocks that stopped growing billions of years ago seems a daunting task. Fortunately, the color and texture of the precipitated minerals record stromatolite growth. The local physical and chemical conditions and the biological makeup of the microbial mat influence the color and the texture of the precipitating minerals [16-19]. Changes in chemistry or microstructure lead to bands of mineral called laminas. Periodic (e.g., seasonal) changes in chemistry and mat microstructure produce a 

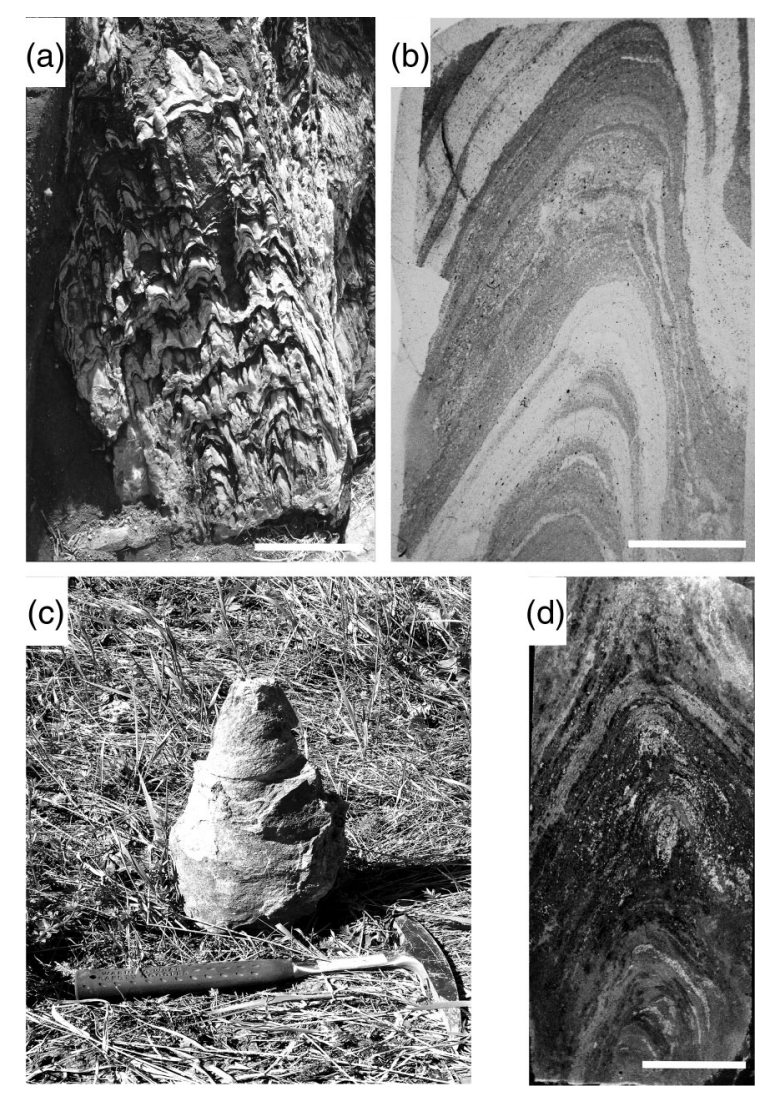

FIG. 1. Conical stromatolites from the Archean and Proterozoic, as seen in the field and in thin sections. (a) A side view of a rock formed by many small conical stromatolites from the Pongola Supergroup [38]. The scale bar is $1 \mathrm{~cm}$. (b) Thin section of a stromatolite from (a). The scale bar is $0.5 \mathrm{~cm}$. (c) Large conical stromatolite from the Bakal Formation [39]. The hammer is approximately $30 \mathrm{~cm}$ long. (d) Thin section of a large cone from the Bakal Formation showing thin bands called laminas. These changes in color and texture record the shape and position of the stromatolite surface at different points in time. The scale bar is $1 \mathrm{~cm}$.

periodic series of laminas. The shape of the resulting bands thus records the position of the growing surface of the stromatolite. The spacing between laminas records the amount of mineral precipitation. This record of stromatolite shape and growth allows one to test growth models [7,20].

The conical form of many stromatolites suggests that they were shaped by a common class of dynamics. To investigate this possibility, we first identify a physical process that may have shaped stromatolites and then develop an idealization of a stromatolite shaped only by this process that is amenable to mathematical analysis. This mathematization has two great advantages. First, it connects qualitative intuition of processes that may shape a stromatolite to a quantitative prediction for the shape of a stromatolite. By comparing this prediction to observations, one can evaluate the utility of the hypothesis. Moreover, and perhaps more importantly, by expressing this hypothesis mathematically, this analysis identifies the set of processes which produce indistinguishable stromatolites.

\section{A HYPOTHESIS AND ITS TEST}

We hypothesize that conical stromatolites are shaped by the diffusive gradients forming within the overlying microbial mat. Most conical stromatolites grew by the precipitation of calcium carbonate within a microbial mat [3]. A priori, it is difficult to know which step in mineral precipitation was rate limiting billions of years ago without knowing the local $p \mathrm{H}$ and concentrations of $\mathrm{Ca}^{2+}$ and inorganic carbon species. However, when mineral precipitation requires the presence of a mat and occurs below the surface of the actively growing biomass, it is reasonable to suspect that the limiting step was the transport of molecules through the mat. Because the relatively inefficient process of molecular diffusion is the primary mechanism of mass transport within a microbial mat [21,22], stromatolite growth was likely limited by the diffusion of some molecule through the microbial mat. This mundane observation has an important implication: Diffusion through a microbial mat couples the growth of precipitated stromatolites to the geometry of the overlying mat.

To test this hypothesis, we reduce stromatolite growth to an analogous physical problem that can be analyzed and understood using techniques from continuum mechanics. As illustrated in Fig. 2(a), in this idealization, a stromatolite grows at a rate proportional to the diffusive flux of a chemical at the base of the mat.

Once idealized, the analysis of a growing stromatolite is guided by a large literature on physical systems in which an interface grows in response to its environment [23-27]. The principal observation that simplifies this analysis is
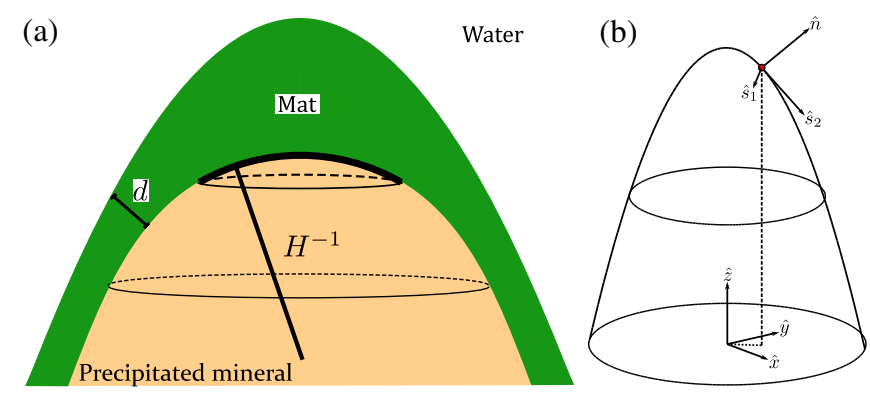

FIG. 2. A schematic of a simplified stromatolite. (a) The stromatolite grows as chemicals diffuse through the microbial mat and precipitate at the base of the mat. The thickness $d$ of the mat varies over the surface of the stromatolite. The shape of the interface between the microbial mat and the stromatolite is described by the local curvature $H$. At the tip of an axisymmetric stromatolite, $H^{-1}$ is the radius of curvature. (b) The transformation from Cartesian coordinates to the local coordinates of the surface $f(x, y)$. The unit vectors $\hat{s}_{1}$ and $\hat{s}_{2}$ are tangent to the surface. The vector $\hat{n}$ is the local normal. The position of the red circle on the stromatolite surface can be expressed in Cartesian coordinates as $\left[x_{0}, y_{0}, f\left(x_{0}, y_{0}\right)\right]$ or in the local coordinates as $\left(s_{1}, s_{2}, 0\right)$, where $s_{1}$ and $s_{2}$ are measured along the stromatolite surface from some known point. For the sake of illustration, we have shown $f$ as a paraboloid. 
that microbial mats are typically thin relative to the size of a stromatolite. As a result, the largest diffusive gradients in the microbial mat are normal to the surface of the stromatolite. The rate at which a stromatolite grows is therefore primarily influenced by processes on the scale of the thickness of the mat.

This insight leads us to hypothesize that the steady-state shape of a stromatolite primarily records the influence of diffusion through a thin film. According to this ansatz, the steady-state shape can be approximated by the translating form predicted by diffusion alone, while the stability of this form may depend on the particular chemical and biological environment. The conical shape predicted by this model is in good quantitative agreement with the shape of precipitated stromatolites as old as $2.9 \times 10^{9}$ yrs.

Moreover, we show that the shape of a stromatolite records the thickness of the former overlying mat. The estimated thickness of stromatolite-forming microbial mats is consistent with modern systems. The agreement between theory and observation leads us to conclude that the conical form of many stromatolites arises from the ubiquitous process of diffusion through a thin film. The fluctuations from this general form may give insight into a specific environmental and biological process shaping a particular stromatolite.

\section{TIME SCALES OF STROMATOLITE GROWTH}

There are three time scales relevant to the growth of a stromatolite. The first is the typical time for the diffusion of small molecules through the mat $\tau_{d}$. For a microbial mat of thickness $d_{0}$, the diffusive time scale is $\tau_{d} \sim d_{0}^{2} / D$, where $D$ is the diffusion coefficient of small molecules or ions in the mat. Taking $D \sim 10^{-6} \mathrm{~cm}^{2} / \mathrm{s}$ [22] and $d_{0} \sim 10^{-1} \mathrm{~cm}$ [28], the diffusive time scale is $\tau_{d} \sim 1 \mathrm{~h}$. The second important time scale is the time $\tau_{g}$ over which the microbial mat grows. Although estimating the doubling time of bacteria billions of years ago is far from trivial, a reasonable guess is between tens of hours and weeks. The third time scale is the rate at which the stromatolite grows by the precipitation of minerals. This time scale is also difficult to estimate in ancient samples, but the rate of mineral precipitation $c_{0}$ can be assumed to be small, compared to the growth of bacteria. In particular, the time scale $\tau_{s}$ over which the stromatolite grows is $\tau_{s} \sim d_{0} / c_{0} \gg \tau_{g}$. This separation of time scales holds if $c_{0} \ll 1 \mathrm{~cm} / \mathrm{yr}$. For comparison, the typical accumulation rate of carbonate platforms in the Phanerozoic is $c_{0} \sim 0.01 \mathrm{~cm} / \mathrm{yr}$ [29].

According to this scaling, stromatolites grow quasistatically. Because $\tau_{d} \ll \tau_{g}$, on the time scale over which a microbial mat grows, the concentration gradients of chemicals in the mat are in the steady state. Similarly, because $\tau_{g} \ll \tau_{s}$, on the time scale over which the stromatolite grows, the shape of the microbial mat is constant in time.

The separation of time scales greatly simplifies the problem of stromatolite growth by breaking growth into three parts. In the next section, we explore how the steadystate concentration of chemicals diffusing through a thin microbial mat is influenced by the shape of the mat. Given this result, we then deduce the rate of mineral precipitation. Finally, we use the resulting growth equation to derive the shape of a stromatolite.

\section{DIFFUSION THROUGH A THIN MICROBIAL MAT}

We shall assume throughout this paper that the rate of mineral precipitation is limited by the diffusion of some chemical through the microbial mat. There are two candidate process that could lead to diffusion-limited growth. First, stromatolite growth could be limited by the diffusive flux of ions related to the precipitation of minerals. Alternatively, the rate of mineral precipitation could be limited by the rate at which the degradation of bacteria provides nuclei for heterogeneous precipitation [30,31]. In this case, the rate of mineral precipitation scales with the turnover time of bacteria living at the base of the microbial mat. If the bacteria are limited by the diffusion of some nutrient through the mat, the turnover time of the population scales with the flux of this nutrient to the base of the mat. In the Appendix, we show how a simple model of mat growth results in increased rates of both growth and degradation where the diffusive flux of nutrients is higher. In both of these cases, the rate of mineral precipitation is expected to scale with a diffusive flux at the base of the mat. In this section, we use the diffusion equation to understand how diffusive transport through a thin mat is affected by the shape of the mat.

According to the diffusion equation, the concentration $\psi$ of a chemical changes in time $t$ according to the equation

$$
\frac{\partial \psi}{\partial t}=D \nabla^{2} \psi
$$

where $D$ is the diffusion coefficient in the microbial mat. As discussed in the previous section, because the time scales over which both the microbial mat and the stromatolite grow are much longer than the diffusive time scale, the concentration of $\psi$ is always in the steady state. Thus, to find the rate at which minerals precipitate, we must solve the equation

$$
\nabla^{2} \psi=0
$$

in the geometry of a microbial mat.

To solve Eq. (2) around a stromatolite, we move to a coordinate system in which the shape of the stromatolite is simply expressed. The transformation to the local coordinate system [32] of the stromatolite is illustrated in Fig. 2(b). In this coordinate system, the basis vectors are $\hat{s}_{1}, \hat{s}_{2}$, and $\hat{n}$. The coordinates $s_{1}$ and $s_{2}$ are distances measured along the surface of the stromatolite in the $\hat{s}_{1}$ and $\hat{s}_{2}$ directions, respectively. The third coordinate $n$ is the distance normal to the surface of the stromatolite. In this 
coordinate system, the surface of the stromatolite is $n=0$ and the surface of the mat is $n=d\left(s_{1}, s_{2}\right)$, where $d\left(s_{1}, s_{2}\right)$ is the local thickness of the mat.

As an elegant and accessible discussion of the Laplacian $\nabla^{2}$ and its representation in curved coordinate systems is presented by Reilly [33], we will simply quote the final result of this coordinate change. Close to the stromatolite surface, Eq. (2) can be expressed as

$$
\frac{\partial^{2} \psi}{\partial n^{2}}+2 H \frac{\partial \psi}{\partial n}+\nabla_{s}^{2} \psi=0
$$

where $H$ is the mean curvature $[32,33]$ of the stromatolite surface. If the shape of a surface near a particular point $p$ is described by the two radii of curvature $R_{1}$ and $R_{2}$, then the mean curvature at $p$ is $H=\left(R_{1}^{-1}+R_{2}^{-1}\right) / 2$. Where $H>0$, the surface of the stromatolite is curved outward (e.g., at the apex of a conical stromatolite). Similarly, regions where $H<0$ are indentations. To make it clear that $H$ varies over the surface, we will simply refer to it as the "curvature." The surface Laplacian $\nabla_{s}^{2} \psi$ describes the flow of $\psi$ parallel to the surface of the stromatolite [33]. For reference, the mean curvature of a sphere of radius $r$ is $H=1 / r$. In this special case, Eq. (3) reduces to the usual representation of the Laplace operator in spherical coordinates.

Because the stromatolite grows in response to molecules diffusing from the surface of the stromatolite to the surface of the mat and vice versa, the largest gradients in concentration are normal to the surface of the stromatolite. To demonstrate this proposition, we rescale every quantity in Eq. (3) by its typical value. Rescaling the concentration $\psi$ by the difference in concentration between the surface of the mat, where $\psi=\psi_{1}$, and the surface of the stromatolite, where $\psi=\psi_{0}$, gives the dimensionless concentration

$$
\phi=\frac{\psi-\psi_{0}}{\psi_{1}-\psi_{0}}
$$

By construction, $\phi=0$ at the surface of the stromatolite and $\phi=1$ at the surface of the mat. Rescaling the curvature $H$ by the typical radius of curvature $R_{0}$ gives the dimensionless curvature

$$
\eta=R_{0} H
$$

Next, we rescale the coordinates $s_{1}, s_{2}$, and $n$ by the typical length scales over which $\psi$ varies. Parallel to the surface, the length scale over which $\psi$ varies is the characteristic size of the stromatolite. We therefore define the dimensionless coordinates

$$
\sigma_{i}=\frac{s_{i}}{R_{0}}
$$

where $i=1,2$. Normal to the surface (i.e., along $\hat{n}$ ), $\psi$ varies over the characteristic thickness of the microbial mat $d_{0}$. Defining the dimensionless normal coordinate $\nu$,

$$
\nu=\frac{n}{d_{0}} .
$$

Reexpressing Eq. (3) in terms of these dimensionless quantities yields

$$
\frac{\partial^{2} \phi}{\partial \nu^{2}}+2\left(\frac{d_{0}}{R_{0}}\right) \eta \frac{\partial \phi}{\partial \nu}+\left(\frac{d_{0}}{R_{0}}\right)^{2} \nabla_{s}^{2} \phi=0 .
$$

Thus, when the microbial mat is thin relative to the size of the stromatolite (i.e., $d_{0} \ll R_{0}$ ), the concentration $\phi$ is a solution to the one-dimensional equation

$$
\frac{\partial^{2} \phi}{\partial \nu^{2}}+2 \epsilon \eta \frac{\partial \phi}{\partial \nu}=0
$$

where $\epsilon=d_{0} / R_{0} \ll 1$.

Physically, Eq. (9) means that the dimensionless concentration and flux of molecules diffusing through a thin microbial mat are only weakly influenced by the macroscopic shape of the mat (e.g., conical). The rate of precipitation is primarily influenced by the local geometry of the mat, as characterized by the dimensionless curvature $\eta$. In the following sections, we will use this result to relate the shape of a stromatolite and the overlying microbial mat to the rate of mineral precipitation.

\section{MICROBIAL-MAT THICKNESS}

To test the central hypothesis of this paper, that stromatolites are shaped by diffusive gradients in the overlying microbial mat, one must relate the geometry of a microbial mat to the shape of the underlying stromatolite.

The focus of this work is on what is common to precipitated stromatolites growing throughout Earth history. Because these stromatolites occurred throughout the last $3.4 \times 10^{9}$ yrs of Earth history - both before and after such basic environmental changes as the rise of oxygen-it is unlikely that any single model of mat growth is appropriate for all precipitated stromatolites. The difficulties in determining an interface growth equation describing the evolution of stromatolites become particularly apparent when one considers the different hydrodynamic environments in which stromatolites grew. Assuming flow velocity of at least several centimeters per second, meter-scale stromatolites grew in turbulent environments. Centimeter-scale stromatolites, by contrast, grew in laminar flows. Because the environments of small and large stromatolites differ qualitatively, it is likely that the dynamics by which an initially flat mat grows small stromatolites differ qualitatively from the dynamics by which meter-scale cones form. Consequently, we take an extremely general model of microbial-mat growth in which to consider the ubiquitous process of diffusion within the mat.

In this section, we assume that there is some nonsingular relationship between the curvature of the mat and the thickness it can grow. For illustrative purposes, an example of how nutrient limitation can give rise to such 
a relationship is included in the Appendix. Although the physical processes shaping a microbial mat depend on the particular environment, it is likely that these processes can be simply expressed. In general, the thickness of a microbial mat is determined by many factors, including the flow of water around the mat [34,35] and the flow of nutrients into the mat [36]. Because these factors depend on the curvature of the mat, it is sensible to suspect that the thickness of a mat is a function of the dimensionless curvature $\epsilon \eta$. Because $\epsilon \ll 1$, the dependence of mat thickness $d(\epsilon \eta)$ on curvature can be approximated by a Taylor series. Thus,

$$
d(\epsilon \eta) \approx d(0)+\left.\frac{\partial d}{\partial \eta}\right|_{\eta=0} \epsilon \eta .
$$

For notational ease, this relation can be expressed as

$$
d=d_{0}\left(1+\epsilon \eta d_{1}\right),
$$

where $d_{0}$ is the thickness of the mat in the absence of curvature and $d_{1}=(\partial d / \partial \eta) / d_{0}$ is the correction to the mat thickness due to curvature.

The generality of the relationship between curvature and mat thickness comes with cost. The particular physical, chemical, and biological processes shaping a microbial mat growing in a particular environment doubtlessly influence the shape and scale of the resulting stromatolite. In the current work, we shall not consider the processes determining the scale of a stromatolite, how the shape of a stromatolite grows from an initially flat mat, or the stability of the resulting form to environmental perturbations. The analysis of these aspects of stromatolite growth requires a more specific experimental characterization of microbial-mat growth that is not available at this point.

\section{GROWTH OF STROMATOLITES}

The solution of the diffusion equation (9) in a microbial mat requires two boundary conditions. Using the dimensionless variables defined in Eqs. (4) and (7), the concentration of the diffusing species reaches a constant value at the base of the mat (i.e., $\nu=0$ )

$$
\phi(0)=0
$$

and at the surface of the mat (i.e., $\nu=1+\epsilon \eta d_{1}$ )

$$
\phi\left(1+\epsilon \eta d_{1}\right)=1 .
$$

Solving Eq. (9) subject to these boundary conditions, we find

$$
\phi(\nu)=\nu+\epsilon \eta\left(1-d_{1}-\nu\right) \nu+\mathcal{O}\left(\epsilon^{2}\right)
$$

Because the rate of mineral precipitation is limited by the rate at which $\phi$ flows to the base of the microbial mat, the flux of $\phi$ at the surface of the stromatolite is proportional to the rate at which minerals precipitate. Thus, the dimensionless velocity $\mathbf{c}$ at which the surface of the stromatolite grows outward is

$$
\mathbf{c}=\left.\frac{\partial \phi}{\partial \nu}\right|_{\nu=0} \hat{n}=\left[1+\left(1-d_{1}\right) \epsilon \eta\right] \hat{n} .
$$

The dimensionless speed $c=\|\mathbf{c}\|$ is measured relative to the rate of mineral precipitation $c_{0}$ in a flat mat. From conservation of mass,

$$
c_{0}=\frac{D}{\rho_{m}} \frac{\psi_{1}-\psi_{0}}{d_{0}},
$$

where $\rho_{m}$ is the concentration of $\psi$ in the precipitated mineral, and, as before, $D$ is the diffusion coefficient of $\psi$ in the microbial mat. Given a value of $d_{1}$, Eq. (15) describes the evolution of a stromatolite surface in time.

This derivation identifies two basic effects that shape stromatolites. The first effect is related to how far chemicals diffuse. Using dimensional variables, the diffusive flux $j$ at the surface of the stromatolite is $j \sim D\left(\psi_{1}-\psi_{0}\right) / d$. Thus, where the overlying mat is thick, mineral precipitation is slow. Consequently, when $d_{1}>0$, the thickening of the mat in regions of high curvature (i.e., $\eta>0$ ) slows the growth of the stromatolite. From Eq. (15), this effect dominates when $d_{1}>1$. The second effect is related to the size of the interface through which chemicals diffuse. In regions of positive curvature, chemicals flow to the stromatolite through a larger mat surface than in regions of negative curvature. This effect causes the stromatolite to grow faster in regions of high curvature. According to Eq. (15), when $0<d_{1}<1$, this effect dominates over the first effect. When $d_{1}<0$, both effects cause regions of positive curvature to grow quickly. As the stromatolite grows, perturbations to the shape of the flat mat vanish if $d_{1}>1$ and grow if $d_{1}<1$. Thus, the stability of a precipitated stromatolite shape depends on how the microbial mat responds to the shape of the underlying surface. The growth of a stromatolite from an initially flat mat, the selection of a particular morphology, and the stability of this form may be affected by lateral diffusion through the mat surface, microbial migration toward favorable regions, and flow of water around the stromatolite. Because these phenomena are neglected in the current formulation and are poorly constrained even in modern microbial mats, model predictions may deviate systematically from observations.

\section{COMPARISON TO ANCIENT STROMATOLITES: LAMINA THICKNESS}

In the previous section, we derived an equation describing the time evolution of the shape of a stromatolite. We now use the geometry of ancient stromatolites to test this model.

Because the lamina records the position of the stromatolite surface through time, the shape and spacing of laminas can be used to test models of stromatolite growth $[7,20]$. If two nearby laminas represent the position of the stromatolite surface at 2 times separated by an amount of time $\delta t$, 

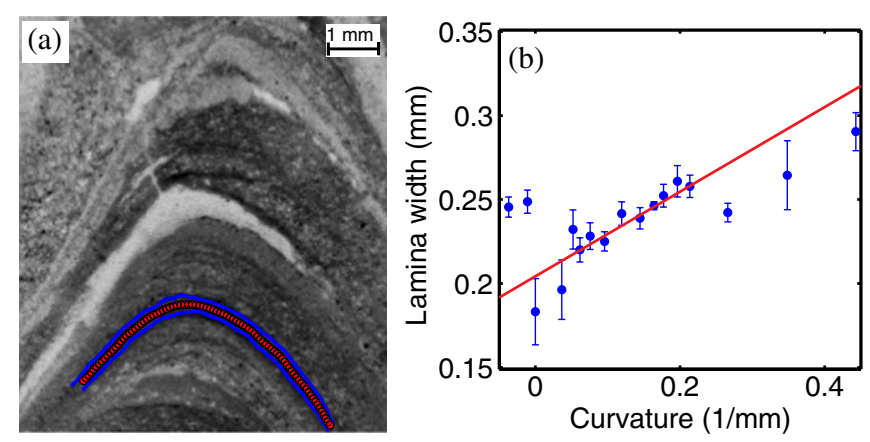

FIG. 3. The shape of stromatolite laminas records the stromatolite's evolution in time. (a) A $2.9 \times 10^{9}$-yr-old conical stromatolite from the Pongola Supergroup in South Africa cut vertically through the center. The transitions between light and dark laminas record the shape of the stromatolite at different points in time. The two solid blue curves represent the surface of the stromatolite at different times. This curve was measured by visually following the transition between a light lamina and a dark lamina. (b) The width of a lamina increases $\left(R^{2}=0.57\right.$, $p<10^{-8}$ ) with curvature. Thus, the rate at which this stromatolite grew is an increasing function of curvature, consistent with Eq. (15). The fit of the measured lamina width and curvature to a straight line gives an estimate of the thickness of the overlying microbial mat $\Delta=1.2 \pm 0.6 \sim 1 \mathrm{~mm}$. Two additional laminas from this sample are shown in the Supplemental Material. Additional curvature-thickness plots are shown in Fig. 5 and the Supplemental Material [41].

then the lamina thickness is $\ell=c \delta t$. Thus, variations in $\ell$ along a lamina record variations in $c$.

To test the growth model proposed in Eq. (15), we measure the thickness and curvature of different points along a lamina. From Eq. (15), the predicted width of a stromatolite lamina is

$$
\ell=c_{0}\left[1+\left(1-d_{1}\right) d_{0} H\right] \delta t .
$$

Thus, the lamina thickness is predicted to increase linearly with lamina curvature. The ratio of the slope of this line to the intercept is $\Delta=\left(1-d_{1}\right) d_{0}$. Assuming that $\left(1-d_{1}\right) \sim$ $1, \Delta$ gives an estimate of the thickness $d_{0}$ of the ancient microbial mat above the lithifying structure.

As shown in Fig. 3(b), lamina thickness is an increasing function of curvature. Fitting these data to Eq. (17), we find that the measured value of $\Delta=1.2 \pm 0.6 \mathrm{~mm}$ is consistent with the thickness $d_{0} \sim 1 \mathrm{~mm}$ of many modern microbial mats [28]. Two observations therefore support the hypothesis of diffusion-limited growth. First, the observed functional dependence of lamina width on curvature is at least qualitatively consistent with the growth Eq. (15). Second, the magnitude of one of the fit parameters $\Delta$ is consistent with the thickness of modern microbial mats [28].

\section{THE SHAPE OF A STROMATOLITE}

Having found that stromatolite laminas grow faster in regions of high curvature, we now ask how this process is recorded in the shape of a stromatolite. In this section, we determine the shape of a rotationally symmetric stromatolite growing at a constant speed $c_{t}$ in the $\hat{z}$ direction under the sole influence of diffusion. This derivation requires an ansatz: The common translating form of a stromatolite most strongly records the ubiquitous process of diffusion through a thin film. While future work may understand the dynamical basis for this assumption, we justify it through comparison to observation.

When the stromatolite grows forward without changing shape, the growth normal to the stromatolite surface balances the growth upward. An illustration of this mode of growth is shown in Fig. 4. If $\theta$ is the angle between the normal vector and the growth direction, then $c=c_{t} \cos \theta$. Substituting this condition for translational growth into Eq. (15) relates the orientation of a point on the stromatolite (i.e., $\theta$ ) to the curvature at that point. In dimensional form, this relation is expressed as

$$
\frac{c_{t}}{c_{0}} \cos \theta=1+\left(1-d_{1}\right) d_{0} H(\theta),
$$

where $H(\theta)$ denotes the dependence of curvature on orientation. This relationship can be reexpressed as a differential equation for the shape of the translating stromatolite. To do so, we describe the surface of the stromatolite as a surface of rotation $f(r)$, where $r$ is the distance of a point on the stromatolite surface from the central axis and $f(r)$ is the height of the stromatolite surface above some reference point. Given these definitions, Eq. (18) becomes

$$
\frac{\chi^{-1}}{\sqrt{1+f^{\prime 2}}}=1-\Delta \frac{r f^{\prime \prime}+f^{\prime}\left(1+f^{\prime 2}\right)}{2 r\left(1+f^{\prime 2}\right)^{3 / 2}},
$$

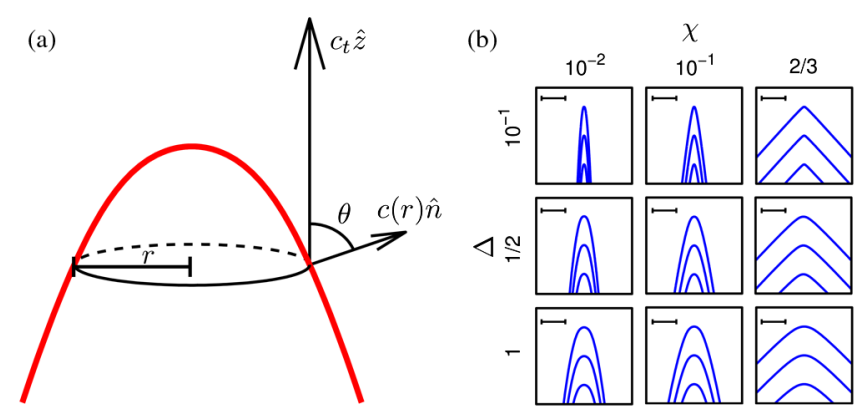

FIG. 4. A balance between curvature-driven growth and translational growth sets the shape of a conical stromatolite. (a) When a curve $f(r)$ (red parabola) evolves due to curvature-driven growth, the normal velocity $c$ is inversely proportional to the mean of the two radii of curvature at that point. When a curve translates forward, there is a geometric relationship between the speed at which a point translates $c_{t}$ and the speed at which it grows in the normal direction $c$. (b) The shape of the stromatolite depends on two parameters. $\chi^{-1}$ is the dimensionless speed of upward growth. The dimensional mat thickness $\Delta$ gives the importance of curvature-driven growth. Values of $\Delta$ are given relative to the thickness of the mat $d_{0}$. The scale bar is $5 d_{0}$. 
where primes represent a derivative with respect to $r$, and $\chi=c_{0} / c_{t}$ is the dimensionless mineral precipitation rate.

To determine the shape of a stromatolite growing under the influence of diffusion, we solve Eq. (19) using the Runge-Kutta method [37]. Because this equation is second order, it requires two boundary conditions which specify the height and slope at the apex. The first boundary condition simply specifies the coordinate system; we take $f(0)=0$. The second boundary condition specifies the slope of the stromatolite near the apex. We consider only smooth stromatolites (i.e., those without singularities in curvature), which requires $f^{\prime}(0)=0$. This constraint is motivated by observations of stromatolites, such as those in Fig. 5, which show that many stromatolites evolved toward a fixed point lacking singularities.
It is important to note that we have not shown that Eq. (15) has a unique solution. Thus, a given stromatolite could potentially grow into a number of distinct forms. It follows from Eq. (19) that, when $f^{\prime}(0)=0, f^{\prime \prime}(r)$ is a continuous function of $f^{\prime}(r)$ and $r$, implying that a unique solution for a rotationally symmetric steadily translating exists. However, it is possible that a greater diversity of steady-state solutions could exist if one relaxes the assumption of a rotational symmetry of the final form. It is possible that certain stromatolites could have grown without ever reaching a translating, steady-state form or found a nonaxisymmetric steady-state form or one with a finite slope at the apex. Comparison of the solution of Eq. (19) to ancient stromatolites-presented in the following section-demonstrates that even if this analysis is limited by assuming that the stromatolite is smooth and
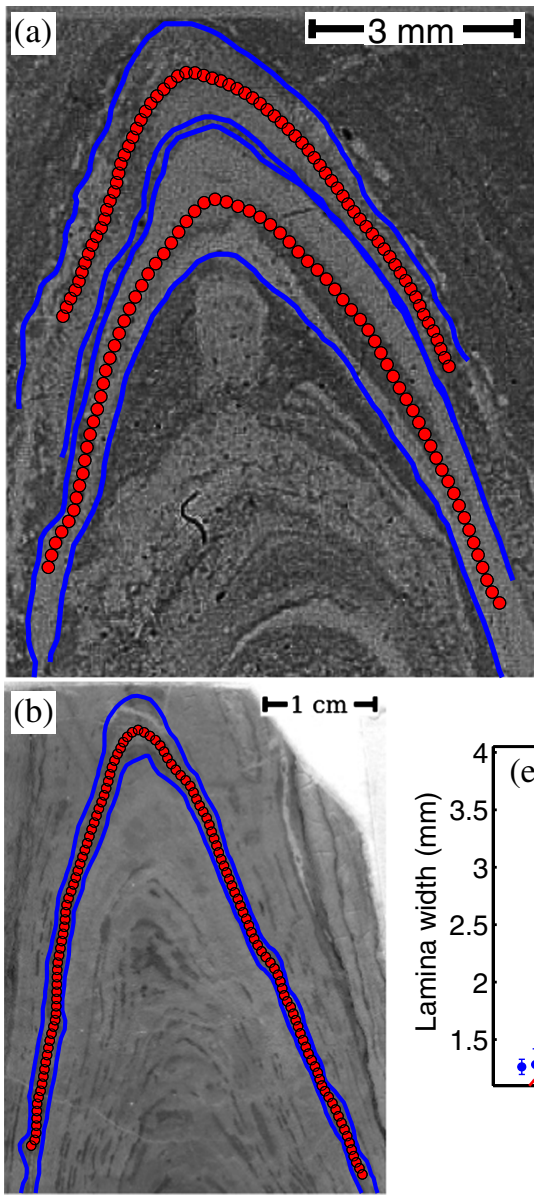
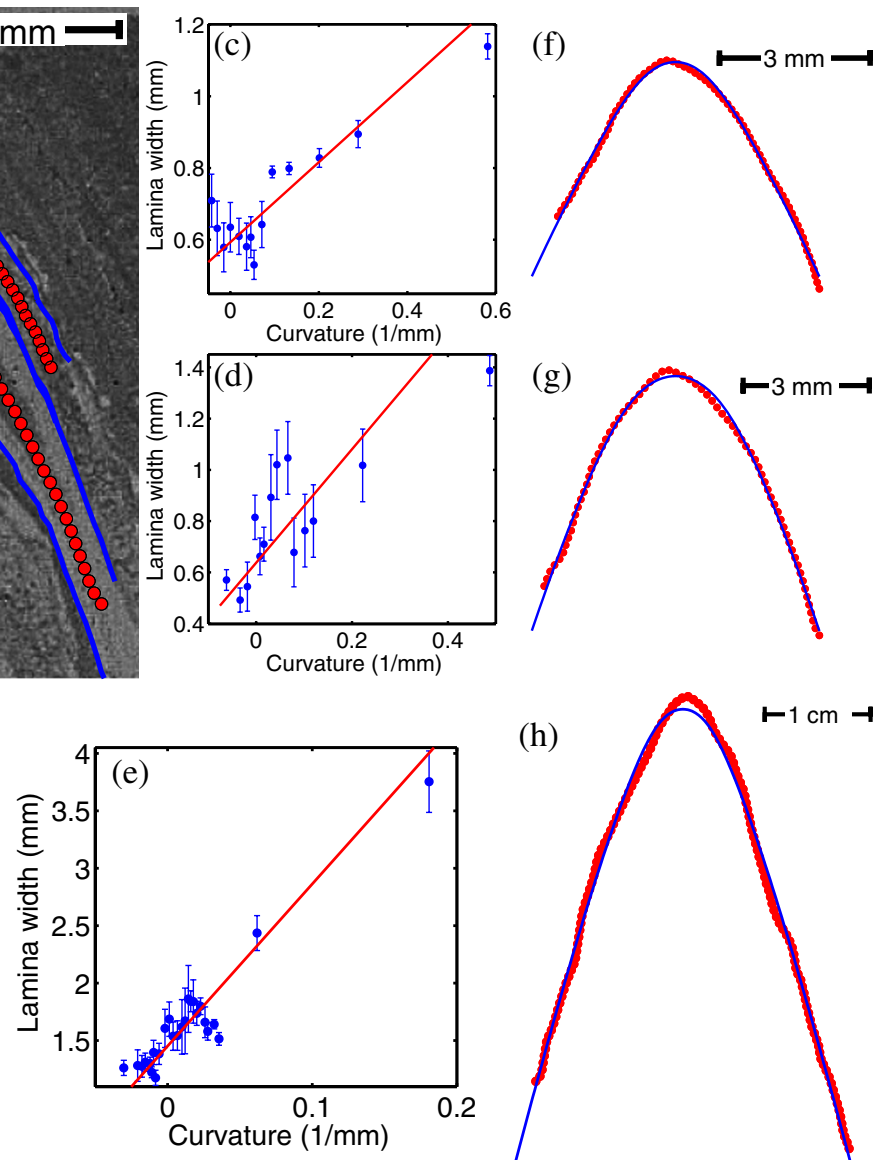

(h)

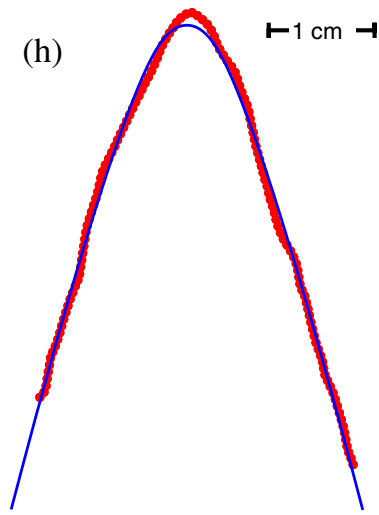

FIG. 5. Comparison of curvature-driven growth to the shapes of large and small conical stromatolites sectioned vertically through the center. (a) Thin section through a $2.9 \times 10^{9}$-yr-old conical stromatolite (photograph) [38]. Solid blue lines represent the upper and lower boundaries of two laminas. The red points lay along a curve equidistant from the traced laminas. (b) Thin section of a $1.6 \times 10^{9}$ yr-old stromatolite from the Bakal Formation [39,40] with traced laminas. (c) The thickness of the upper laminas from (a) increases with lamina curvature $\left(R^{2}=0.81, p<10^{-17}, \Delta=1.9 \pm 0.7 \mathrm{~mm}\right)$, consistent with Eq. (15). (d) Similar agreement is found for the lower lamina $\left(R^{2}=0.64, p<10^{-8}, \Delta=3.5 \pm 1.7 \mathrm{~mm}\right)$ and in the (e) Proterozoic sample shown in $(\mathrm{b})\left(R^{2}=0.88, p<10^{-44}\right.$, $\Delta=9.7 \pm 1.6 \mathrm{~mm}$ ). (f),(g) Fitting two parameters, the measured shapes of the (f) upper and (g) lower laminas (points) are consistent with the shape (solid curve) of a lamina growing by the curvature-driven growth equation (19). (f) Fit of the sample from (b) to Eq. (19). All samples are cut through the central axis of the cone. 
axisymmetric, stromatolites consistent with this assumption are represented in the rock record.

As shown in Fig. 4, the translating shape of a stromatolite growing by mineral precipitation is qualitatively similar to the shape of conical stromatolites. Two physical processes influence the shape: mineral precipitation, as characterized by $\chi$, and diffusive gradients within the microbial mat, as characterized by $\Delta$. The conical shape of a translating stromatolite represents a balance between these two processes. An understanding of the shape follows from two observations. First, where the surface is sharply curved, diffusive gradients cause minerals to precipitate quickly. Second, where the stromatolite is steeply sloped, a little bit of mineral precipitation normal to the surface causes a large amount of vertical growth. Thus, if a stromatolite is to retain its shape while growing vertically at a constant speed, it must be relatively steep where it is less curved. Because the stromatolite is smooth and symmetric, at the center zone, $f^{\prime}=0$. Thus, near $r=0$, Eq. (19) can be approximated as $f^{\prime \prime}=2(\chi-1) / \Delta \chi=$ const. The apex of a translating stromatolite is therefore a paraboloid, the curvature of which is a decreasing function of $\Delta$. All else being equal, thinner mats should form more sharply curved stromatolites. Far from the central axis, the curvature becomes negligible. To compensate for the resulting decrease in the mineral precipitation rate, the side walls must be steeper. Far from the central axis of the stromatolite, Eq. (19) can be approximated as $f^{\prime 2}=\left(1-\chi^{2}\right) / \chi^{2}=$ const. Thus, far from the apex, the sides of the stromatolite become straight lines. Combining the paraboloid apex with the asymptotically straight side-wall limits, the translating shape of a stromatolite has straight walls that curve to form the apex. This balance gives the conical shape, where the slope of the walls far from the apex records the rate of mineral precipitation relative to the speed of upward growth and the curvature at the apex records the thickness of the microbial mat.

\section{COMPARISON TO ANCIENT STROMATOLITES: LAMINA SHAPE}

The predictions of Eq. (19) can be quantitatively compared to the laminas of conical stromatolites. Here, we use two examples: a small cone from the $2.9 \times 10^{9}$-yrold Pongola Supergroup [38] and a considerably larger cone from the approximately $1.5 \times 10^{9}$-yr-old Bakal Formation [39,40]. In either case, we characterize the shape of a lamina by averaging the curves that mark the upper and lower boundaries of the lamina, as described in Sec. XI. As shown in Fig. 5, the lamina shape is in close agreement with the two-parameter model described in Eq. (19). The comparisons of theory and observation for five additional samples [40] from these formations are shown in the Supplemental Material [41].

As discussed in the previous section, the shape of the overlying microbial mat affects the shape of the resulting lamina. In particular, one of the fit parameters in Eq. (19) is the estimated thickness of the ancient microbial mat $\Delta$, the same parameter estimated in Sec. VII. In Fig. 3, this parameter is estimated from a comparison of thickness of a lamina to the curvature. In this section, $\Delta$ is estimated from the overall shape of the lamina (the red curve in Fig. 5), without reference to the lamina thickness. To distinguish between these estimates, $\Delta_{t}$ is the thickness of the mat estimated from the relation between lamina thickness and curvature. In contrast, $\Delta_{s}$ is the thickness estimated from the shape of the curve, assuming that the shape of the stromatolite is constant. Because these two estimates rely on different measurements and assumptions, the comparison of these estimates provides an important self-consistency check of the curvature-driven growth model.

As shown in Fig. 6, the estimates of mat thickness by the two methods produce consistent results. Variations between the estimates beyond the statistical uncertainty reflect the influence of two types of error. First, because the two estimates of mat thickness rely on different approximations, deviations from these approximations are reflected in systematic errors in the estimated mat thickness. In particular, the estimate of $\Delta_{s}$ assumes that the stromatolite translates forward at a constant rate without changing form. In fact, the shape generally changes slightly between neighboring laminas. This small change gives corrections to the shape of the stromatolite which

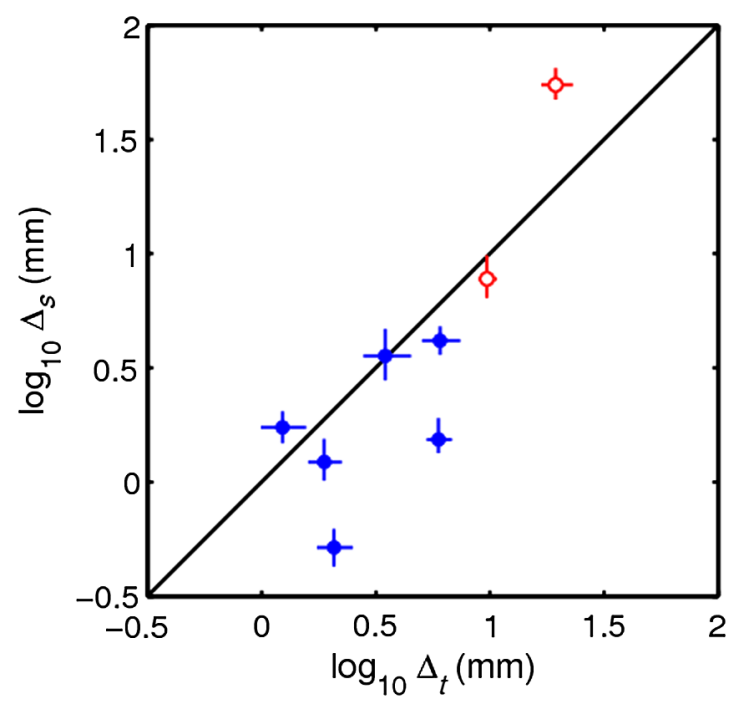

FIG. 6. Two estimates of the thicknesses of ancient stromatolite-forming microbial mats produce consistent values. $\Delta_{t}$ is estimated from the relation between the curvature of a lamina and the distance to the neighboring laminas. $\Delta_{s}$ is estimated from the shape of a single lamina. The black line shows equality. The measured slope is $m=2.3 \pm 1.6$, consistent with the predicted value $m=1$. The intercept $b<0$ reflects that $\Delta_{t}$ is systematically larger than the estimate of $\Delta_{t}$. Solid blue points represent stromatolites from the $2.9 \times 10^{9}$-yr-old Pongola Formation [38]. Open red points are from Proterozoic samples. 
influence the estimate of $\Delta_{s}$. The second source of error arises from processes that may weakly influence the growth of stromatolites such as the flow of water around the mat and the migration of microbes along the mat. Although these processes are not necessary to explain the basic conical shape of the stromatolite, they may influence the precise form. Ignoring these processes may introduce small, but systematic, errors in the estimate of $\Delta$.

We have found that the observed width and shape of stromatolite laminas are consistent with curvature-driven growth, and the two estimates of microbial-mat thickness are broadly consistent. We conclude that the basic conical form of many stromatolites records the influence of diffusion.

\section{DISCUSSION AND CONCLUSION}

The central hypothesis of this paper is that the influence of diffusion through a thin film is apparent in the shapes of precipitated stromatolites. Diffusive gradients, extending over the thickness of the microbial mat, cause mineral precipitation to be faster in regions of high curvature. Because the typical length scale of this effect is the thickness of the mat, the shape of a precipitated stromatolite records the thickness of the overlying microbial mat. Comparing the thickness of a stromatolite lamina to the curvature, we find that growth is faster in regions of high curvature. Moreover, the shape of a stromatolite growing upward under the influence of diffusion alone is consistent with the observed shapes of conical stromatolites. Both of these tests support the hypothesis that diffusion through a thin film shaped stromatolites throughout Earth history.

Past studies of stromatolite morphogenesis have considered two roles for diffusion [42]. In the first, lateral diffusion of sediment $[7,20]$ is included along with surfacenormal and vertical growth in the dynamics of stromatolite growth. In the second, sediment grains are randomly advected by turbulent flows and become stuck when they encounter a stromatolite $[3,43]$; the resulting growth is an example of diffusion-limited aggregation [44]. In this paper, we consider a new role for diffusion. We show how diffusive gradients within a microbial mat shape the resulting stromatolite.

Most models of stromatolite morphology begin by using physical reasoning to motivate a particular growth model. This model is then shown to reproduce observations. With few exceptions [45], the parametrization of these models or the comparison to data has made it difficult to compare model parameters with modern systems. Consequently, comparisons to observations are a much stronger test of the functional form of the growth dynamics than of the model interpretation. To gain a better interpretation of fossils, we have adopted a slightly different approach. We begin by showing how diffusive transport through a microbial mat relates the shape of the underlying stromatolite to its growth and resulting form. By relating the shape to a particular physical process, our results can be simply compared to modern systems. In particular, the inferred thickness of ancient stromatolite-forming microbial mats is consistent with their modern counterparts.

The contribution of this paper is in understanding the influence of diffusion alone upon the shape of stromatolites. Because diffusion through a thin film is common to all precipitated stromatolites, its influence must be understood before one infers the influence of biological and environmental factors.

Although diffusion through a thin film is ubiquitous, it is certainly not the only process that shapes stromatolites. Indeed, curvature-driven growth alone is not sufficient to understand the growth of an initially flat mat into a conical stromatolite. Because this growth equation favors the growth of all small, highly curved perturbations, these dynamics cause an initially flat mat to grow into an increasingly rough surface. In reality, lateral diffusionneglected in Eq. (8) — smoothes out bumps that form on the scale of the microbial-mat thickness. Larger perturbations are likely smoothed by the material properties of the microbial mat and the behavior of microbes. For example, because microbial mats cannot physically support arbitrarily steep features [45] and because phototaxis or growth toward light causes bacteria to move out of shaded areas, overhanging features may be selected against in photosynthetic mats. Because diffusion through a thin microbial mat is common to many stromatolites, it is reasonable to suspect that this process strongly influences the steadystate shape of a stromatolite. We find that this suspicion is supported by observation.

Our results lead us to conclude that the conical shape of a precipitated stromatolite is a general feature of a class of dynamics. While a record of evolutionary innovations (e.g., photosynthesis) and environmental revolutions (e.g., oxygenation of the atmosphere) may be found in the precise variations in stromatolite form, the basic conical shape is a poor indicator. Notably, this analysis identifies a deep similarity between the growth of stromatolites and the growth of stalactites [26] and icicles [46]. In both of these systems, the interface grows in response to diffusion through a thin film of water. Despite the dramatically different environments, stromatolites, stalactites, and icicles all share a similar conical shape. The shapes of stalactites and icicles are modified from the form predicted by Eq. (15) by the flow of water over the surface.

Although the basic conical form of stromatolites is consistent with diffusion through a film, the comparison between any particular lamina and model predictions shows substantial variability. The variability records the particular biological (e.g., phototaxis) and environmental (e.g., flow conditions) conditions that shaped ancient microbial mats. Thus, the conditions shaping a particular stromatolite may be most easily seen in their nonequilibrium growth 
and the deviations of the observed equilibrium shape from the shape predicted by diffusion alone.

We now briefly review the characteristics of a microbial mat that are recorded in the basic conical shape. We then speculate on how certain biological and environmental processes may be recognized in a more detailed analysis of stromatolite form. Finally, we outline how a systematic investigation of the shapes of stromatolites may reveal additional biological and environmental processes that shape stromatolites.

This study identifies two roles of the microbial mat in shaping the stromatolite. First, the microbial mat prevents water from flowing immediately around the precipitated surface of the stromatolite, leading to the formation of the diffusive gradients throughout the mat. The microbial mat acts as a diffusive boundary layer around the stromatolite. The typical thickness of this layer $d_{0} \sim 1 \mathrm{~mm}$. By way of comparison, the thickness $\delta_{\mu}$ of the boundary layer in the absence of a microbial mat is the length scale on which viscosity balances inertia. If water flows around a stromatolite of height $h$ at a speed $u$, then the thickness of the boundary layer is determined by the scaling $\rho u^{2} / h \sim$ $\mu u / \delta_{\mu}^{2}$, where $\rho$ is the density of water and $\mu$ is the viscosity. For a viscous boundary layer of thickness $\delta_{\mu} \sim 1 \mathrm{~mm}$ around a stromatolite of height $h=1 \mathrm{~cm}$, the flow must be $u \sim 1 \mathrm{~cm} / \mathrm{s}$. Microbial mats may effectively increase the thickness of the boundary layer.

Second, the microbial mat determines the time scale over which the stromatolite grows. From Eq. (16), the characteristic rate of mineralization is $c_{0} \propto D / d_{0}$, where the dimensionless proportionality coefficient depends on the local chemistry and may be small. This observation informs the interpretation of lamina thickness. Stromatolite laminas are thought to record the periodic cycles of growth in a microbial mat and differences in the arrangement of cells in the microbial mat $[5,10]$. Because the arrangement of cells in a mat may change both $D$ and $d_{0}$, differences in lamina thickness record differences in the parameters describing the microbial mat. For example, if $D$ is nearly constant in a microbial mat, then the ratio of lamina thicknesses records the ratio of mat thicknesses or cell densities. Future work would benefit greatly from a detailed understanding of how $D$ and $d_{0}$ change in response to ecological and physiological variations in the mat.

We now briefly discuss how the shape of a stromatolite may be modified by two phenomena. Our derivation assumes that the water around the stromatolite is well mixed. When stromatolites grow in still water [11], all of the basic phenomena of curvature-driven growth remain; however, they must be coupled to diffusion around the mat. Similarly, interactions between distant parts of a microbial mat can be mediated by an anisotropic flow. For example, variations in the flow of water over the complex shape of a biofilm can lead to systematic variations in the thickness of the overlying diffusive boundary layer [47]. To take this phenomenon into account, one must couple the diffusion within the mat to advection and diffusion around the mat. This effect is currently only considered for certain heterotrophic biofilms [48]. The coupling of mineral precipitation to mat growth and the advection and diffusion of chemicals in the surrounding water can also influence the growth of the stromatolite in response to environmental perturbations.

Another phenomenon that may have influenced the shape of ancient conical stromatolites is the growth of the microbial mat toward light $[6,8]$. To include this process, one must understand how the average thickness of a microbial mat is affected by photosynthesis and phototaxis. One intuitive possibility is that a microbial mat is thicker where the light intensity is higher. Thus, Eq. (11) may be modified to include additional terms that depend on the local slope of the stromatolite. Both of these corrections may apply to some large stromatolites that grew in deeper, subtidal settings, in the presence of weak currents [49].

To understand the diversity of stromatolite forms seen in the fossil record, one must develop a quantitative description of processes other than diffusion that shaped stromatolites. Two avenues of inquiry appear particularly attractive. First, laboratory experiments can cast light on the processes shaping modern microbial mats that form precipitated stromatolites. In the simplest case, one might measure how the shape of the mat surface changes as the stromatolite grows. By combining these measurements with models of nutrient flow, one may develop a quantitative understanding of the relationship between the growth of microbes and the growth of precipitated stromatolites in different hydrodynamic environments. Second, the transient evolution of stromatolite morphology is recorded in the shapes of preserved laminas. Growth in a randomly fluctuating environment gives rise to small perturbations in the shape of the stromatolite. The growth or decay of these perturbations records the small-scale dynamics shaping the stromatolite. It may be possible to extract the average influence of fluid flow and the growth of the microbial mat from the evolution of these perturbations.

By expressing the hypothesis that stromatolites are shaped by diffusive gradients mathematically, this derivation identifies a class of biological, physical, and environmental processes that produce indistinguishable forms. Equation (15) was derived by considering the leadingorder influence of curvature on a diffusive flux, but its functional form is more general. Whenever the rate of mineral precipitation is coupled to diffusion through a thin film, variations in the rate of mineral precipitation can be expanded in powers of the small parameter $d_{0} H$, thus yielding Eq. (15). Practically, this observation means that the conical shape of a stromatolite does not record any single biological or environmental process but rather a geometric feature common to a class of processes. 
In conclusion, we have found that diffusion-limited growth connects mat form to stromatolite growth. The comparison of the observed shape of laminas to the predictions of curvature-driven growth validates this hypothesis. The conical morphology of a stromatolite identifies it with a family of dynamical systems defined by diffusion through a thin film. Because the conical morphology is common to a class of biological and environmental processes, one cannot infer from shape alone the signature of any single biological or environmental process. Future interpretations of conical stromatolites should combine this result with additional textural and sedimentological information to develop an understanding of the processes shaping ancient systems. By recognizing the shape of a conical stromatolite in a general, but precise, mathematical form, this result identifies the dynamical basis for the ubiquity of conical stromatolites throughout Earth history.

\section{MATERIALS: SELECTION OF LAMINAS}

The lamina thickness and curvature are measured by tracing the boundaries of a lamina. Laminas are traced from thin sections of stromatolites that are cut vertically through the central axis. Laminas are traced by eye from the transition in color between a light band a dark band. In practice, few laminas are sufficiently well preserved that the transition between these bands can be traced for more than a few millimeters. Moreover, because this analysis requires both the curvature and thickness of a lamina, the data are restricted to those laminas with clearly visible upper and lower boundaries. Consequently, a single thin section generally provides only a single usable lamina, and no sample produced more than three laminas.

Because the estimate of curvature requires estimating the second derivative of a curve, estimates of the curvature are very sensitive to noise. To reduce the influence of measurement noise, two laminas are averaged by finding the curve passing exactly midway between the two traced laminas. To do so, the distance of each point between the two laminas from either lamina is measured. The average lamina is then taken as the curve traced by the points that are at a maximum distance from the boundaries. The lamina thickness is measured normal to the averaged lamina. For example, if the boundaries of two laminas are the lines $y= \pm 1$, the point $\left(x_{0}, y_{0}\right)$ is a distance $\ell_{m}=1-\left|y_{0}\right|$ from the boundaries. Because $\ell_{m}$ reaches its maximum at $y_{0}=0$, the shape of the average lamina is $y_{a}=0$.

Recalling that $H$ is the curvature of the surface, its value depends on the three-dimensional shape of the lamina. To take into account the curvature of the lamina around the apex, we approximate the stromatolite as rotationally symmetric. Given this approximation, the curvature

$$
H=\frac{r f^{\prime \prime}+f^{\prime}\left(1+f^{\prime 2}\right)}{2 r\left(1+f^{\prime 2}\right)^{3 / 2}},
$$

where $r$ is the radial distance of a point on the lamina from the central axis, and $f(r)$ is the traced shape of the lamina. The uncertainties in the estimates of lamina thickness and curvature are estimated from the distribution of widths measured for points with similar curvatures.

\section{ACKNOWLEDGMENTS}

We would like to thank the MIT Geomicrobiology Lab, M. S. Sim, O. Devauchelle, J. Friedman, D. Forney, and C. Follett for helpful suggestions and discussions. This work was supported by NASA Grant No. NNA08CN84A, NSF Grant No. EAR-0420592, and the Solomon Buchsbaum Fund.

\section{APPENDIX: DYNAMICS OF BIOFILM GROWTH}

This Appendix presents a derivation of the relationship between the thickness of a nutrient-limited microbial mat and the shape (Fig. 7). For the sake of simplicity, we assume that the growth rate is

$$
g(k)=A\left(k-k_{0}\right),
$$

where $k$ is the concentration of the limiting nutrient, $k_{0}$ is the minimum concentration needed for cell growth, and $A$ is a proportionality constant. At points in the mat where $k>k_{0}$, the mat grows. Where $k<k_{0}$, there is degradation. It is straightforward to adapt this derivation to incorporate a more realistic growth equation. Because the results of this derivation are not compared to observations, we use this qualitatively correct model because it is analytically tractable.

The equilibrium thickness of a mat is the thickness at which degradation balances growth. This condition is expressed mathematically by the integral condition

$$
G(d)=\int_{0}^{d} g(k) d n=0 .
$$

Thus, to find the thickness of a microbial mat, we must first solve for the nutrient profile through a mat.

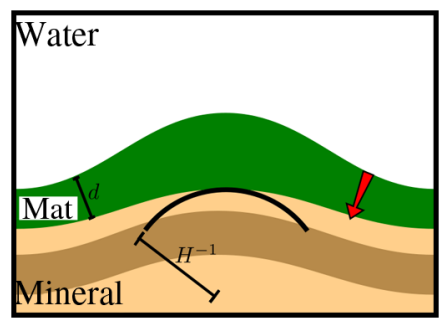

FIG. 7. A toy model of mat growth. Nutrients (arrow) diffuse through a microbial mat and are consumed. Near the nutrientrich mat surface, the mat grows. At the nutrient-poor mat bottom, the mat decays. As described in Eq. (A15), mat thickness is determined by the balance of growth and decay. The equilibrium thickness varies with the curvature of the underlying stromatolite. 
Nutrients flow through a microbial mat by diffusion $[21,22]$. As a nutrient flows through the mat, it is consumed by the constituent microbes. The balance between nutrient diffusion and uptake in modern stromatolite-forming microbial mats is accurately described by the equation

$$
\nabla^{2} k=\frac{k}{\lambda^{2}},
$$

where $\lambda$ is the typical distance into the mat the nutrient diffuses before being consumed [50]. This equation is solved subject to two boundary conditions. At the surface of the mat $(n=d)$, the concentration reaches a constant value

$$
k(d)=k_{\infty} .
$$

At the base of the mat $(n=0)$, there is no flow of limiting nutrient into the underlying stromatolite

$$
\left.\frac{\partial k}{\partial n}\right|_{n=0}=0 .
$$

Given a solution of $k$ through the mat, Eq. (A2) gives the net growth rate of the mat.

To solve for the nutrient profile in the mat, we first nondimensionalize the model. As in the derivation of Eq. (9), the flow of nutrients simplifies considerably in a thin film. Expanding Eq. (A3), we find

$$
\frac{\partial^{2} \xi}{\partial \tilde{\nu}^{2}}+2\left(\frac{\lambda}{R_{0}}\right) \eta \frac{\partial \xi}{\partial \tilde{\nu}}+\left(\frac{\lambda}{R_{0}}\right)^{2} \nabla_{s}^{2} \xi=\xi,
$$

where the dimensionless coordinate $\tilde{\nu}=n / \lambda$, and the dimensionless nutrient concentration $\xi=k / k_{\infty}$. Identifying the small parameter $\tilde{\epsilon}=\lambda / R_{0}$, Eq. (A6) becomes the one-dimensional equation

$$
\frac{\partial^{2} \xi}{\partial \tilde{\nu}^{2}}+2 \tilde{\epsilon} \eta \frac{\partial \xi}{\partial \tilde{\nu}}=\xi
$$

Solving for $\xi$, subject to the boundary conditions (A4) and (A5), we find

$$
\xi(\tilde{\nu})=\frac{\cosh \tilde{\nu}}{\cosh \tilde{\delta}}+\tilde{\epsilon} \eta \frac{\tilde{\delta} \cosh \tilde{\nu}+\sinh \tilde{\nu}-\tilde{\nu} \cosh \tilde{\nu} \tanh \tilde{\delta}}{\cosh \tilde{\delta}}
$$

where the dimensionless mat thickness is $\tilde{\delta}=d / \lambda$.

Given this solution of the nutrient profile, we now calculate the growth rate of the mat. Nondimensionalizing Eq. (A2), the dimensionless growth rate of the mat is

$$
\Gamma(\tilde{\delta})=\frac{G}{k_{\infty} \lambda A}=\int_{0}^{\tilde{\delta}}\left[\xi(\tilde{\nu})-\xi_{0}\right] d \tilde{\nu},
$$

where $\xi_{0}=k_{0} / k_{\infty}$. Integrating, we find

$$
\Gamma(\tilde{\delta})=-\xi_{0} \tilde{\delta}+\tanh \tilde{\delta}+\tilde{\epsilon} \eta\left(2-2 \operatorname{sech} \tilde{\delta}-\tanh ^{2} \tilde{\delta}\right) .
$$

From this equation, we find that when $\tilde{\delta} \ll 1, \Gamma=$ $\left(1-\xi_{0}\right) \tilde{\delta}>0$. Physically, this result means that in a thin mat, nutrients permeate the community, causing the mat to grow. When $\tilde{\delta} \gg 1, \Gamma=-\xi_{0} \tilde{\delta}<0$, causing the mat to degrade and thus grow thinner. In this limit, nutrients only penetrate into the surface of the mat. Thus, in the majority of the mat, $\xi<\xi_{0}$, causing the mat to degrade. There is, therefore, an intermediate thickness of the mat at which the growth rate of microbes at the surface exactly balances the rate at which the mat is degraded. This intermediate point gives the equilibrium thickness of a microbial mat. To find the leading-order dependence of mat thickness on curvature, we find the coefficients $\tilde{\delta}_{0}$ and $\tilde{\delta}_{1}$, such that

$$
\Gamma\left(\tilde{\delta}_{0}+\tilde{\epsilon} \eta \tilde{\delta}_{1}\right)=0+\mathcal{O}\left(\tilde{\epsilon}^{2}\right) .
$$

Expanding Eq. (A10), we find that the characteristic thickness of a microbial mat $\tilde{\delta}_{0}$ is a solution of the equation

$$
\xi_{0}=\frac{\tanh \tilde{\delta}_{0}}{\tilde{\delta}_{0}}
$$

Moreover, the correction to the mat thickness due to curvature is given by the coefficient

$$
\tilde{\delta}_{1}=\frac{8 \tilde{\delta}_{0} \sinh ^{4}\left(\tilde{\delta}_{0} / 2\right)}{\sinh \left(2 \tilde{\delta}_{0}\right)-2 \tilde{\delta}_{0}} .
$$

Equivalently, in dimensional form, the characteristic thickness of the mat $d_{0}$ is related to the minimal concentration of nutrients necessary for growth through the equation

$$
\frac{k_{0}}{k_{\infty}}=\frac{\lambda \tanh \left(d_{0} / \lambda\right)}{d_{0}} .
$$

Thus, the thickness of the mat is

$$
d=d_{0}\left(1+\lambda H \tilde{d}_{1}\right)
$$

where, according to this model, the dimensionless coefficient

$$
\tilde{d}_{1}=\frac{8 \sinh ^{4}\left(d_{0} / 2 \lambda\right)}{\sinh \left(2 d_{0} / \lambda\right)-2 d_{0} / \lambda} .
$$

Because $d_{1}>0$, the mat grows thicker in regions of high curvature. Because the thickness of the mat is constant, this increase in growth rate must be balanced by increased degradation deeper in the mat.

[1] A. C. Allwood, M. R. Walter, B. S. Kamber, C. P. Marshall, and I.W. Burch, Stromatolite Reef from the Early Archaean Era of Australia, Nature (London) 441, 714 (2006).

[2] H. J. Hofmann, K. Grey, A. H. Hickman, and R. I. Thorpe, Origin of 3.45 Ga Coniform Stromatolites in Warrawoona Group, Western Australia, Geol. Soc. Am. Bull. 111, 1256 (1999).

[3] J.P. Grotzinger and A.H. Knoll, Stromatolites in Precambrian Carbonates: Evolutionary Mileposts or 
Environmental Dipsticks?, Annu. Rev. Earth Planet Sci. 27, 313 (1999).

[4] E. Kalkowsky, Oolith und Stromatolith im norddeutschen Buntsandstein (Oolites and Stromatolites in North German Buntsandstein), Zeitschrift der Deutschen Geologischen Gesellschaft 60, 68 (1908).

[5] M. R. Walter, in Earth's Earliest Biosphere: Its Origin and Evolution, edited by J. W. Schopf (Princeton University Press, Princeton, NJ, 1983), p. 187-213.

[6] M. R. Walter, J. Bauld, and T. D. Brock, in Stromatolites, edited by M. R. Walter (Elsevier, Amsterdam, 1976), p. $273-310$.

[7] J. P. Grotzinger and D. H. Rothman, An Abiotic Model for Stromatolite Morphogenesis, Nature (London) 383, 423 (1996).

[8] M. T. Batchelor, R. V. Burne, B. I. Henry, and S. D. Watt, Deterministic KPZ Model for Stromatolite Laminae, Physica (Amsterdam) 282A, 123 (2000).

[9] M. T. Batchelor, R. V. Burne, B. I. Henry, and M.J. Jackson, A Case for Biotic Morphogenesis of Coniform Stromatolites, Physica (Amsterdam) 337A, 319 (2004).

[10] T. Bosak, B. Liang, M.S. Sim, and A.P. Petroff, Morphological Record of Oxygenic Photosynthesis in Conical Stromatolites, Proc. Natl. Acad. Sci. U.S.A. 106, 10939 (2009).

[11] A. P. Petroff, M. S. Sim, A. Maslov, M. Krupenin, D. H. Rothman, and T. Bosak, Biophysical Basis for the Geometry of Conical Stromatolites, Proc. Natl. Acad. Sci. U.S.A. 107, 9956 (2010).

[12] A. Martin, E. G. Nisbet, and M.J. Bickle, Archean Stromatolites of the Belingwe Greenstone Belt, Zimbabwe (Rhodesia), Precambrian Res. 13, 337 (1980).

[13] H. J. Hofmann and M. Masson, Archean Stromatolites from Abitibi Greenstone Belt, Quebec, Canada, Bull. Geol. Soc. Am. 106, 424 (1994).

[14] T. D. Brock, in Thermophilic Microorganisms and Life at High Temperatures (Springer-Verlag, New York, 1978), p. 337-385.

[15] B. Jones, R. W. Renaut, M. R. Rosen, and K. M. Ansdell, Coniform Stromatolites from Geothermal Systems, North Island, New Zealand, Palaios 17, 84 (2002).

[16] C. DeFarge, J. Trichet, A.-M. Jaunet, M. Robert, J. Tribble, and F.J. Sansone, Texture of Microbial Sediments Revealed by Cryo-scanning Electron Microscopy, J. Sediment. Res. 66, 935 (1996).

[17] P. Gautret, G. Camoin, S. Golubic, and S. Sprachta, Biochemical Control of Calcium Carbonate Precipitation in Modern Lagoonal Microbialites, Tikehau Atoll, French Polynesia, J. Sediment. Res. 74, 462 (2004).

[18] T. Bosak, J. W. M. Bush, M. R. Flynn, B. Liang, S. Ono, A. P. Petroff, and M.S. Sim, Formation and Stability of Oxygen-Rich Bubbles that Shape Photosynthetic Mats, Geobiology 8, 45 (2010).

[19] M. Rogerson, H. M. Pedley, and R. Middleton, Microbial Influence on Macroenvironment Chemical Conditions in Alkaline (Tufa) Streams: Perspectives from In Vitro Experiments, Geological Society Special Publication 336, 65 (2010).

[20] M. T. Batchelor, R. V. Burne, B. I. Henry, and S. D. Watt, Mathematical and Image Analysis of Stromatolite Morphogenesis, Math. Geol. 35, 789 (2003).
[21] D. de Beer, P. Stoodley, and Z. Lewandowski, Measurement of Local Diffusion Coefficients in Biofilms by Microinjection and Confocal Microscopy, Biotechnol. Bioeng. 53, 151 (1997).

[22] P. S. Stewart, Diffusion in Biofilms, J. Bacteriol. 185, 1485 (2003).

[23] P. G. Saffman and G. I. Taylor, The Penetration of a Fluid into a Porous Medium or Hele-Shaw Cell Containing a More Viscous Liquid, Proc. R. Soc. A 245, 312 (1958).

[24] W. W. Mullins and R. F. Sekerka, Morphological Stability of a Particle Growing by Diffusion or Heat Flow, J. Appl. Phys. 34, 323 (1963).

[25] P. Pelcé, Dynamics of Curved Fronts (Academic, New York, 1988).

[26] M. B. Short, J. C. Baygents, and R. E. Goldstein, Stalactite Growth as a Free-Boundary Problem, Phys. Fluids 17, 083101 (2005).

[27] J. Veysey II and N. Goldenfeld, Watching Rocks Grow, Nat. Phys. 4, 310 (2008).

[28] D. de Beer and M. Kühl, in The Benthic Boundary Layer: Transport Processes and Biogeochemistry (Oxford University Press, New York, 2001), p. 374-389.

[29] H. Bosscher and W. Schlager, Accumulation Rates of Carbonate Platforms, J. Geol. 101, 345 (1993).

[30] T. Bosak and D. K. Newman, Microbial Nucleation of Calcium Carbonate in the Precambrian, Geology 31, 577 (2003).

[31] C. Dupraz, R. P. Reid, O. Braissant, A. W. Decho, R. S. Norman, and P.T. Visscher, Processes of Carbonate Precipitation in Modern Microbial Mats, Earth-Sci. Rev. 96, 141 (2009).

[32] R.S. Millman and G. D. Parker, Elements of Differential Geometry (Prentice-Hall, Englewood Cliffs, NJ, 1977).

[33] R. C. Reilly, Mean Curvature, the Laplacian, and Soap Bubbles, Am. Math. Mon. 89, 180 (1982).

[34] B. E. Rittman, The Effect of Shear Stress on Biofilm Loss Rate, Biotechnol. Bioeng. 24, 501 (1982).

[35] M. O. Pereira, M. Kuehn, S. Wuertz, T. Neu, and L.F. Melo, Effect of Flow Regime on the Architecture of a Pseudomonas fluorescens Biofilm, Biotechnol. Bioeng. 78, 164 (2002).

[36] B. E. Rittmann and P.L. McCarty, Model of SteadyState-Biofilm Kinetics, Biotechnol. Bioeng. 22, 2343 (1980).

[37] J.R. Dormand and P.J. Prince, A Family of Embedded Runge-Kutta Formulae, J. Comput. Appl. Math. 6, 19 (1980).

[38] N. J. Beukes and D. R. Lowe, Environmental Control on Diverse Stromatolite Morphologies in the $3000 \mathrm{Myr}$ Pongola Supergroup, South Africa, Sedimentology 36, 383 (1989).

[39] A. B. Kuznetsov, G. V. Ovchinnikova, I. M. Gorokhov, O. K. Kaurova, V. N. Krupenin, and A. V. Maslov, SrIsotope Signature and $\mathrm{Pb}-\mathrm{Pb}$ Age of the Bakal Formation Limestones in the Lower Riphean Type Section, the Southern Urals, Doklady of the Academy of Sciences of the USSR Earth Sciences Sections 391, 819 (2003).

[40] F. Y. Vlasov, in Materialy po paleontologii srednego paleozoya Urala $i$ Sibiri. Sverdlovsk (Notes on the Paleontology of the Middle Paleozoic of the Urals and Siberia), edited by M. E. Raaben (Akademiya Nauk SSSR, 
Uralskii nauchnii tzentr; Trudy instituta geologii i geokhimii, 1977), p. 101-128.

[41] See Supplemental Material at http://link.aps.org/ supplemental/10.1103/PhysRevX.3.041012 for additional comparisons between the shape of stromatolites and model predictions.

[42] Tanja Bosak, Andrew H. Knoll, and Alexander P. Petroff, The Meaning of Stromatolites, Annu. Rev. Earth Planet Sci. 41, 21 (2013).

[43] C. Dupraz, R. Pattisina, and E. P. Verrecchia, Translation of Energy into Morphology: Simulation of Stromatolite Morphospace Using a Stochastic Model, Sedimentary Geology 185, 185 (2006).

[44] T. A. Witten, Jr., and L. M. Sander, Diffusion-Limited Aggregation, a Kinetic Critical Phenomenon, Phys. Rev. Lett. 47, 1400 (1981).

[45] M. M. Tice, D.C.O. Thornton, M.C. Pope, T.D. Olszewski, and J. Gong, Archean Microbial Mat Communities, Annu. Rev. Earth Planet Sci. 39, 297 (2011).
[46] M. B. Short, J. C. Baygents, and R. E. Goldstein, A FreeBoundary Theory for the Shape of the Ideal Dripping Icicle, Phys. Fluids 18, 083101 (2006).

[47] B. B. Jørgensen and D. J. Des Marais, The Diffusive Boundary Layer of Sediments: Oxygen Microgradients over a Microbial Mat, Limnol. Oceanogr. 35, 1343 (1990).

[48] M.C.M. Van Loosdrecht, J.J. Heijnen, H. Eberl, J. Kreft, and C. Picioreanu, Mathematical Modelling of Biofilm Structures, Antonie van Leeuwenhoek 81, 245 (2002).

[49] J. Bertrand-Sarfati and A. Moussine-Pouchkine, Evolution and Environmental Conditions of ConophytonJacutophyton Associations in the Atar Dolomite (Upper Proterozoic, Mauritania), Precambrian Res. 29, 207 (1985).

[50] Alexander P. Petroff, Ting-Di Wu, Biqing Liang, Jeannie Mui, Jean-Luc Guerquin-Kern, Hojatollah Vali, Daniel H. Rothman, and Tanja Bosak, Reaction-Diffusion Model of Nutrient Uptake in a Biofilm: Theory and Experiment, J. Theor. Biol. 289, 90 (2011). 